\title{
Inlet Trade Study for a Low-Boom Aircraft Demonstrator
}

Christopher M. Heath \& John W. Slater

NASA GRC - Cleveland, OH

Sriram K. Rallabhandi

National Institute of Aerospace - Hampton, VA

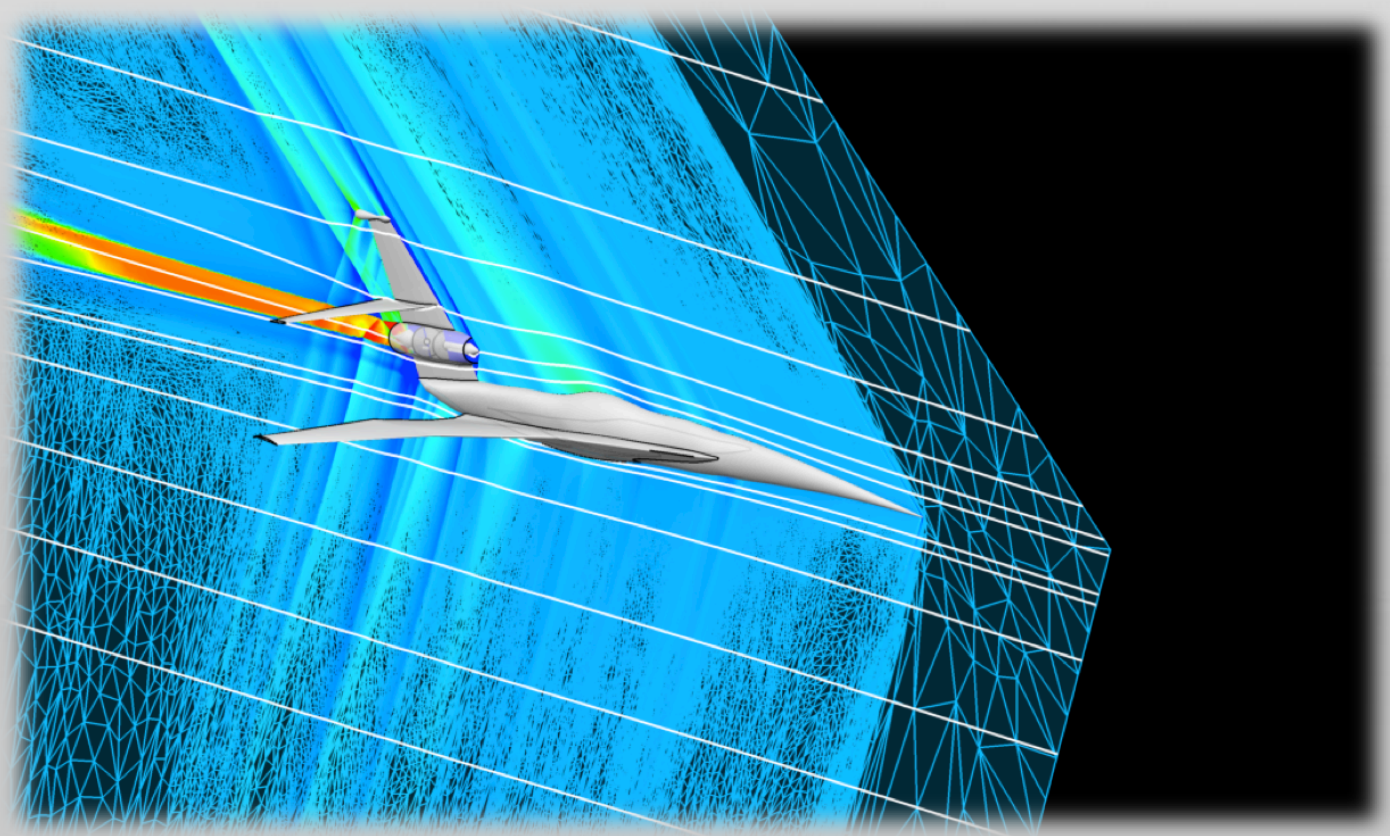

\section{AIAA Aviation Conference Washington, D.C. June 13-17, 2016}




\section{Research Motivation}

Overland sonic boom challenges supersonic aircraft viability.

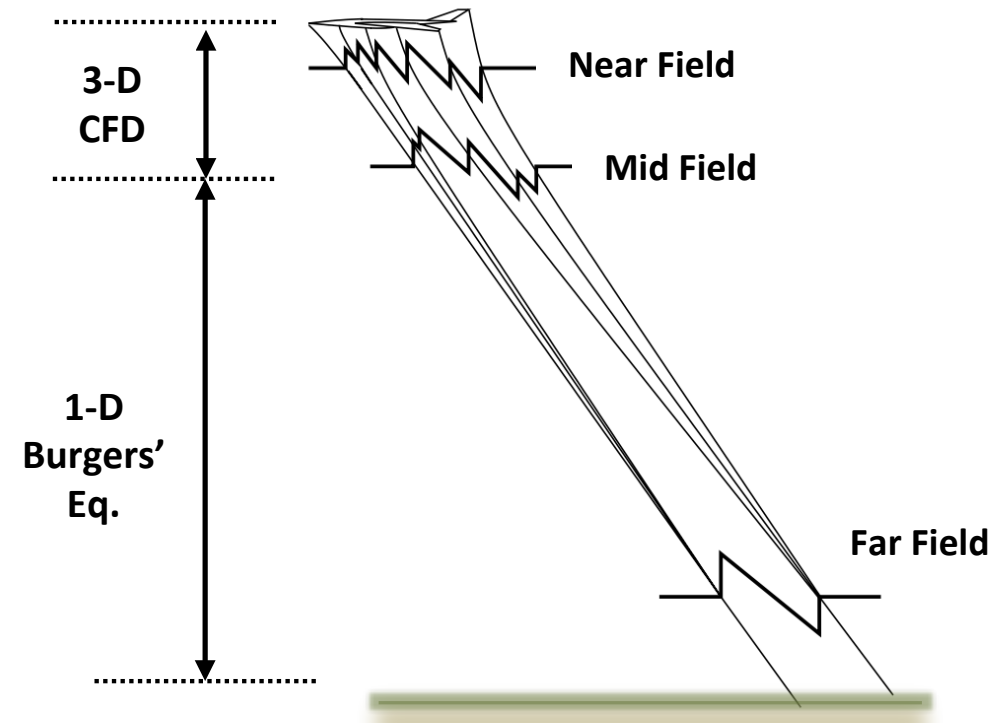

\section{Current State-of-the-Art:}

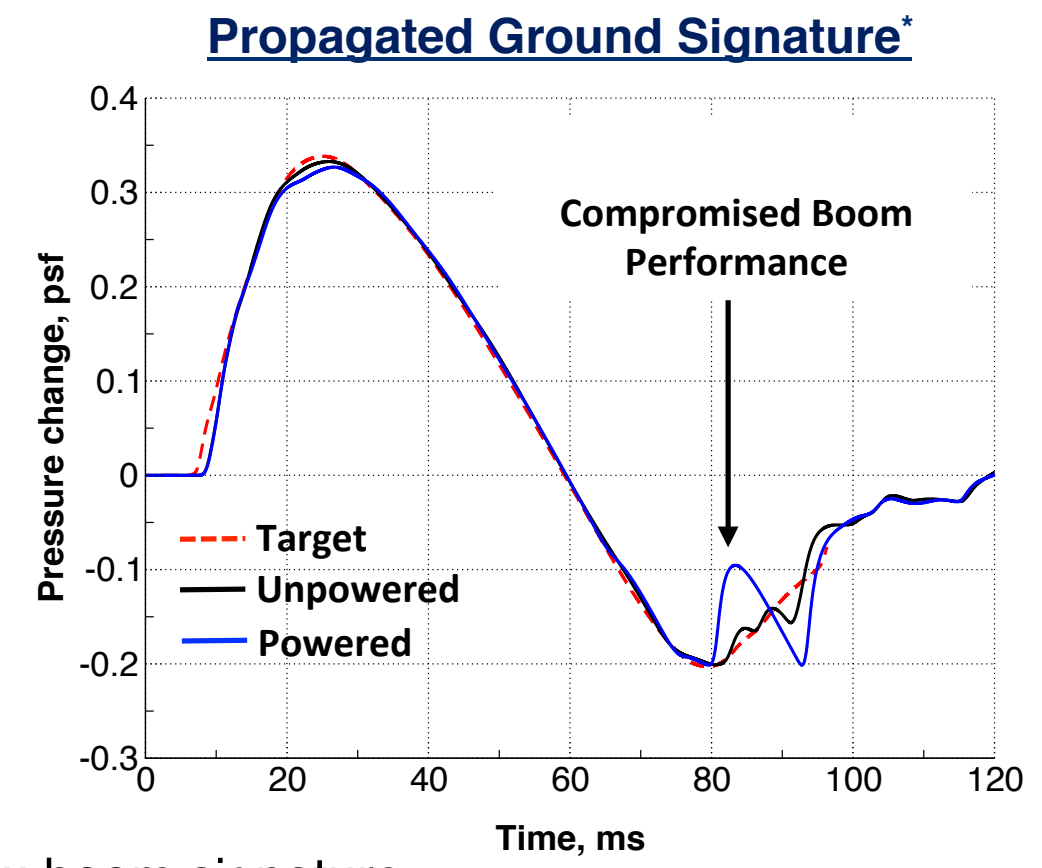

Propagated Ground Signature*

- Adjoint-based shape optimization to match low-boom signature

- Isolated inlet, engine core, nozzle design and subsequent integration

Drawbacks:

- Low-boom optimization neglects propulsion effects, sacrifices inlet/airframe performance \& TSFC to meet low-boom objective

- Research shows introducing propulsion effects into a pre-optimized airframe pressure signature can compromise low-boom performance

*Wintzer, M. et. al., AIAA Paper No. 2015-1045. 


\section{Research Objectives}

1. Quantify installation effects on inlet/engine performance.

2. Quantify installation effects on airframe/sonic boom performance.

\section{Approach:}

Compare isolated vs. installed performance of two inlet types on aerodynamically tailored low-boom reference* airframe.

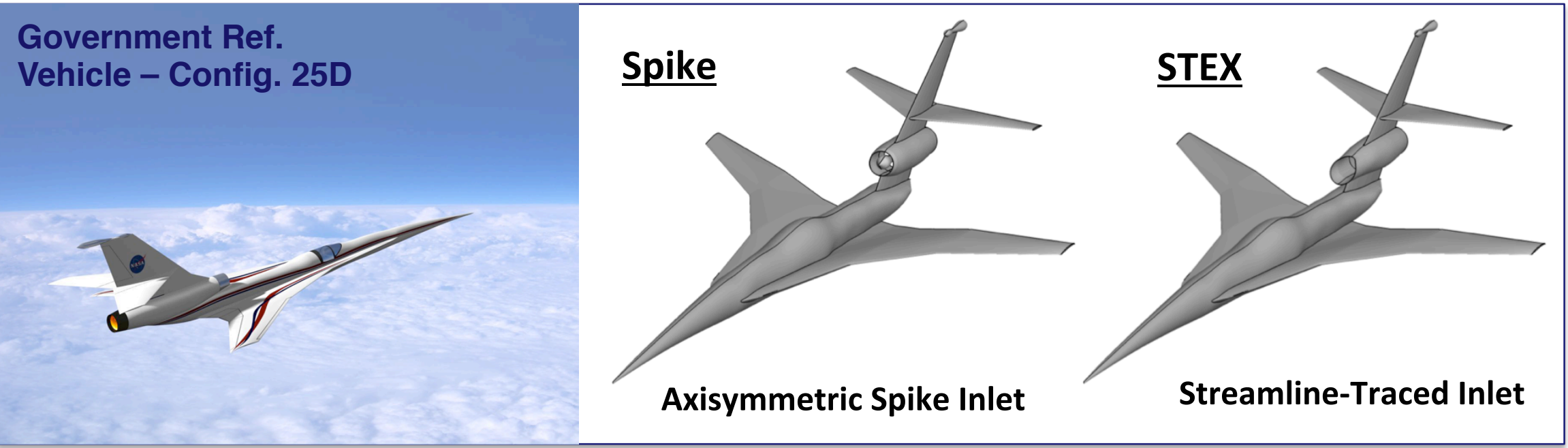

${ }^{*}$ Ref. vehicle designed w/Euler adjoint-based shape optimization to achieve under-track loudness <76.4 PLdB. Wintzer, M. et. al., AIAA Paper No. 2015-1045. 


\section{Problem Definition - Single Pt. Design}

\section{Reference Cruise Pt.}

- 55K-ft std. day alt.

- Mach no. $=1.6$

- $\mathrm{C}_{\mathrm{L}}=0.065, \mathrm{a} \approx 3.25^{\circ}$

- $21 \mathrm{~K} \mathrm{lb}$ cruise weight

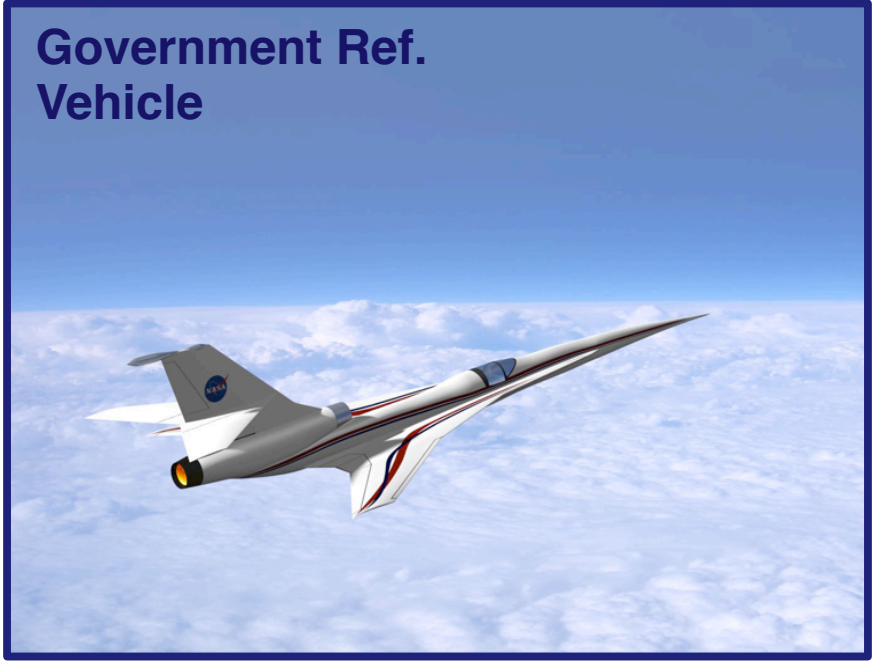

\begin{tabular}{|l|}
$\begin{array}{l}\text { Estimated Reference } \\
\text { GE-F404-402 Conditions }\end{array}$ \\
$\underline{\text { Inlet }}$ \\
- $\mathrm{W}_{2}=51.2-\mathrm{lbm} / \mathrm{s}$ \\
- $\mathrm{P}_{\mathrm{t}, 2}=6.1-\mathrm{psi}$ \\
- $\mathrm{T}_{\mathrm{t}, 2}=590-\mathrm{deg} \mathrm{R}$ \\
Nozzle \\
- $\mathrm{W}_{6}=52.6-\mathrm{lbm} / \mathrm{s}$ \\
- $\mathrm{P}_{\mathrm{t}, 6}=21.4-\mathrm{psi}$ \\
- $\mathrm{T}_{\mathrm{t}, 6}=2852-\mathrm{deg} \mathrm{R}$ \\
- $\mathrm{TSFC}=1.53-\mathrm{lbm} / \mathrm{lbf}-\mathrm{hr}$ \\
- $\mathrm{F}_{\mathrm{net}}=4487-\mathrm{lbf}$ \\
\hline
\end{tabular}




\section{Solution Overview}

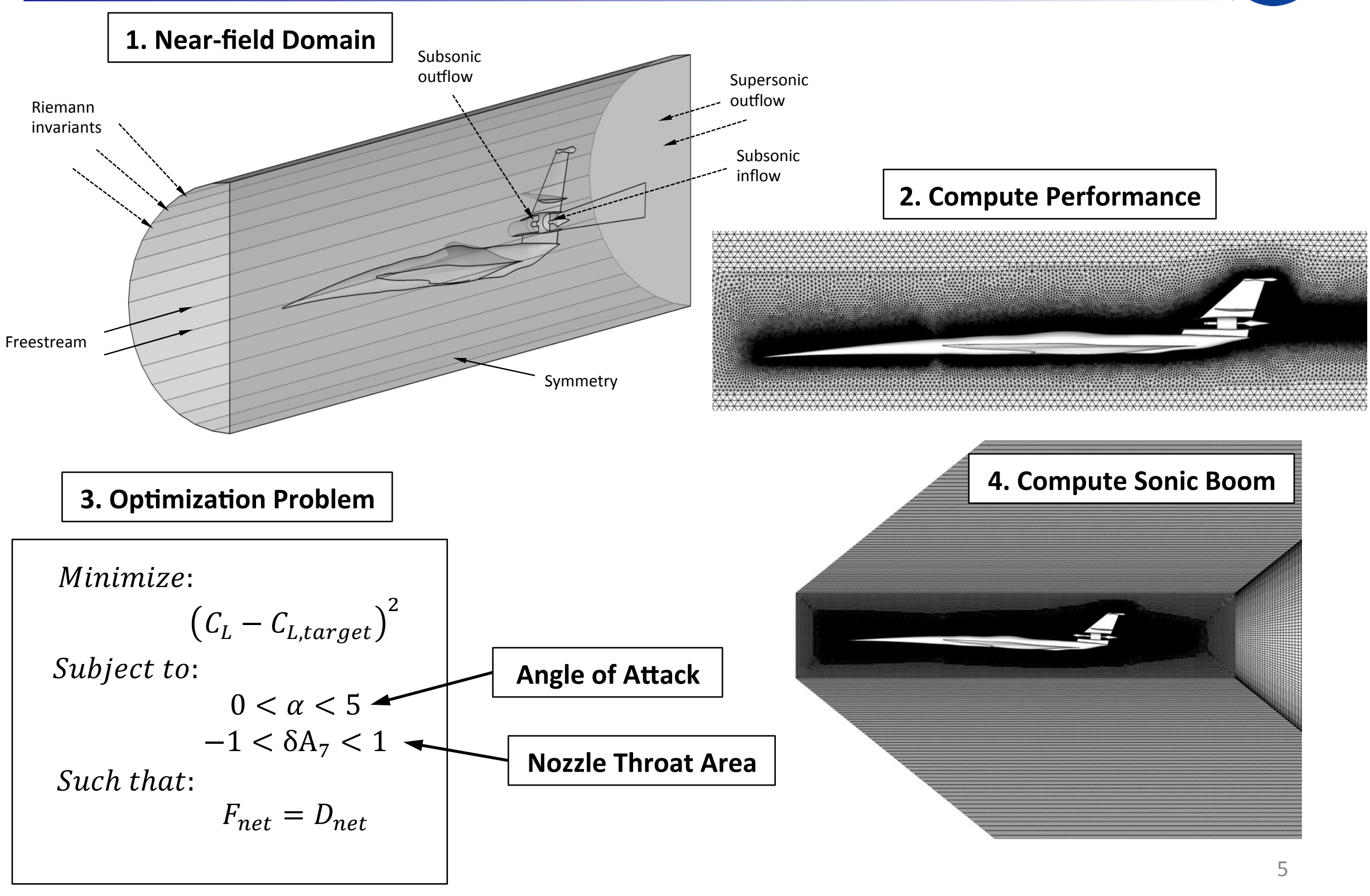




\section{Computational Steps}

1. Parameterize airframe geometry (ESP).

2. Design \& size custom inlets (SUPIN).

3. Integrate inlet/airframe geometry (ESP).

4. Discretize surface geometry (Pointwise).

5. Discretize volume w/plume sourcing (AFLR3).

6. Compute RANS vehicle performance (Fun3D).

7. Compute inlet rec. \& adjust ref. engine cycle. (NPSS)

8. Balance vehicle forces using adjoint-based optimization (Fun3D/SNOPT).

9. Generate sonic boom grid (Inflate).

10. Perform sonic boom RANS analysis (Fun3D).

11. Extrapolate mid-field signatures to ground and convert to perceived loudness (sBOOM). 


\section{Inlet Performance Comparison}

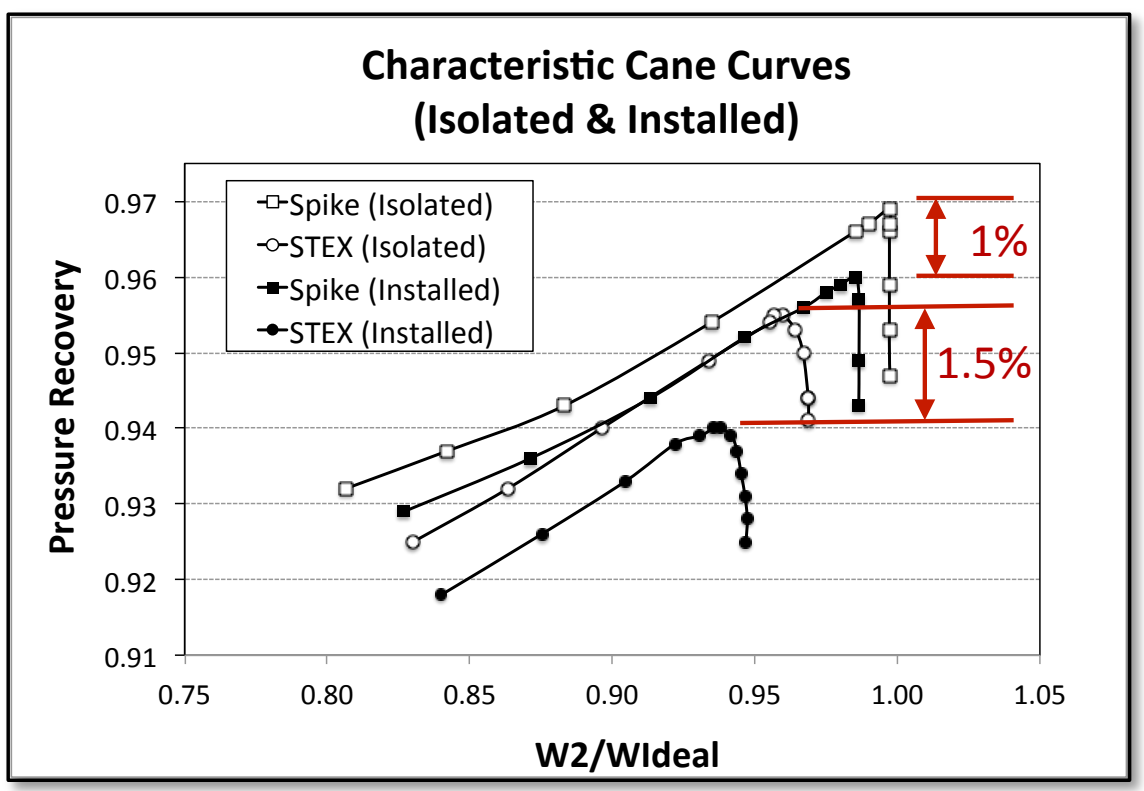

Similar isolated/installed characteristics

\section{Installed Spike}

- Peak recovery declines by $\sim 1 \%$

- $\sim 1 \%$ reduction in mass flow rate

\section{Installed STEX}

- Peak recovery declines by $\sim 1.5 \%$

- $\sim 2 \%$ reduction in mass flow rate
Isolated

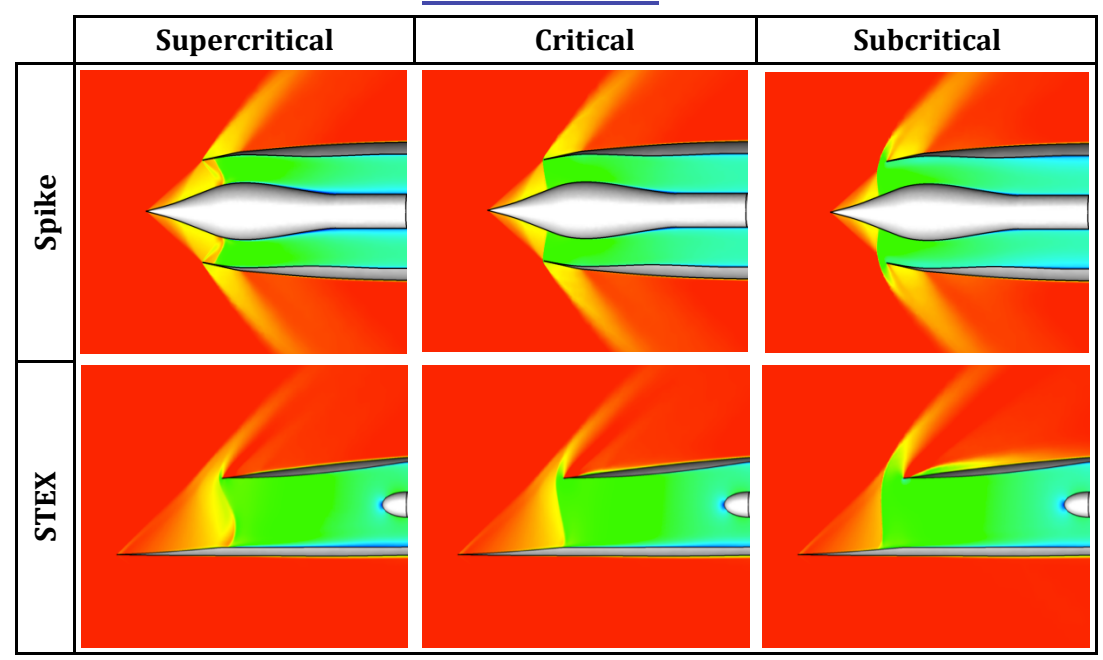

Installed

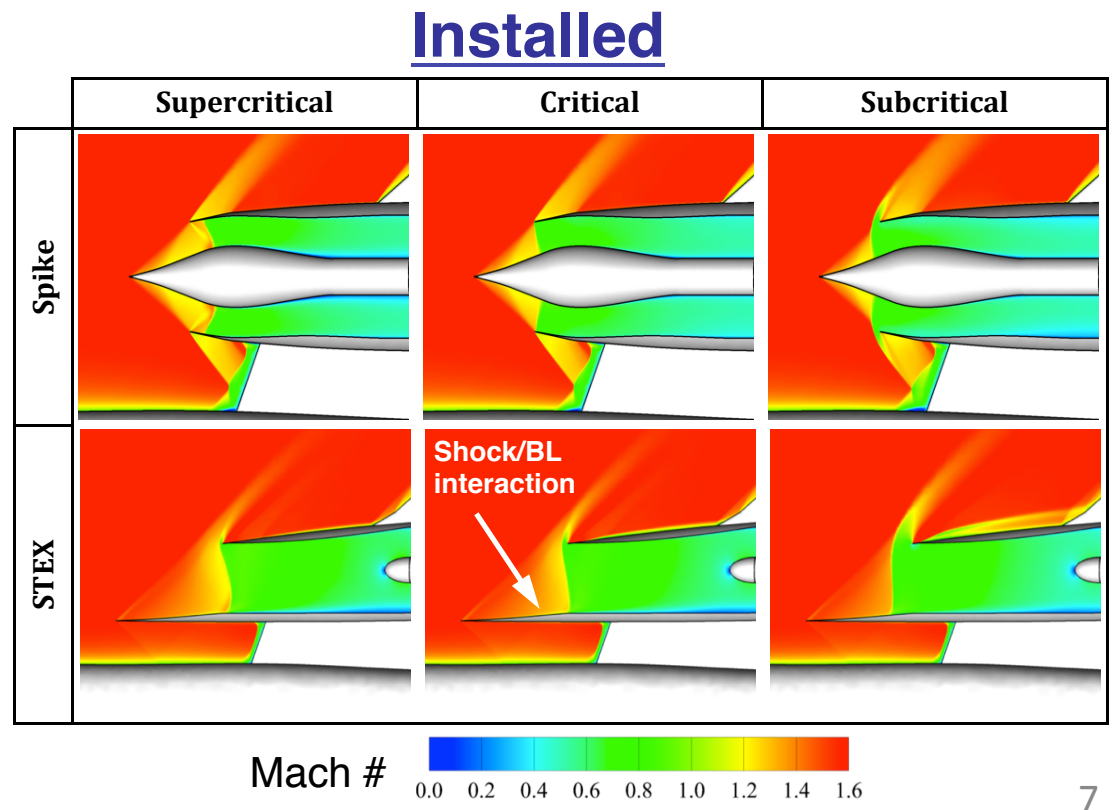

$\begin{array}{lllllllllllll} & \text { Mach \# } & & & & & & & & & & \\ & 0.0 & 0.2 & 0.4 & 0.6 & 0.8 & 1.0 & 1.2 & 1.4 & 1.6\end{array}$ 


\section{Inlet Performance Comparison @ AIP}

\begin{tabular}{|c|c|c|}
\hline Parameter & STEX & Spike \\
\hline $\mathrm{P}_{\mathrm{t}, 2} / \mathrm{P}_{\mathrm{t}, 0}$ & 0.94 & 0.97 \\
\hline $\mathrm{DPC} / \mathrm{P}$ & 0.0408 & 0.0075 \\
\hline $\mathrm{DPR} / \mathrm{P}$ & 0.086 & 0.028 \\
\hline
\end{tabular}

- Installed spike inlet recovery 3\% higher than STEX recovery

- Both inlets meet SAE ARP radial \& circumferential distortion requirements for GE-F404-402

- Spike inlet fan distortion at AIP is significantly lower than STEX inlet distortion
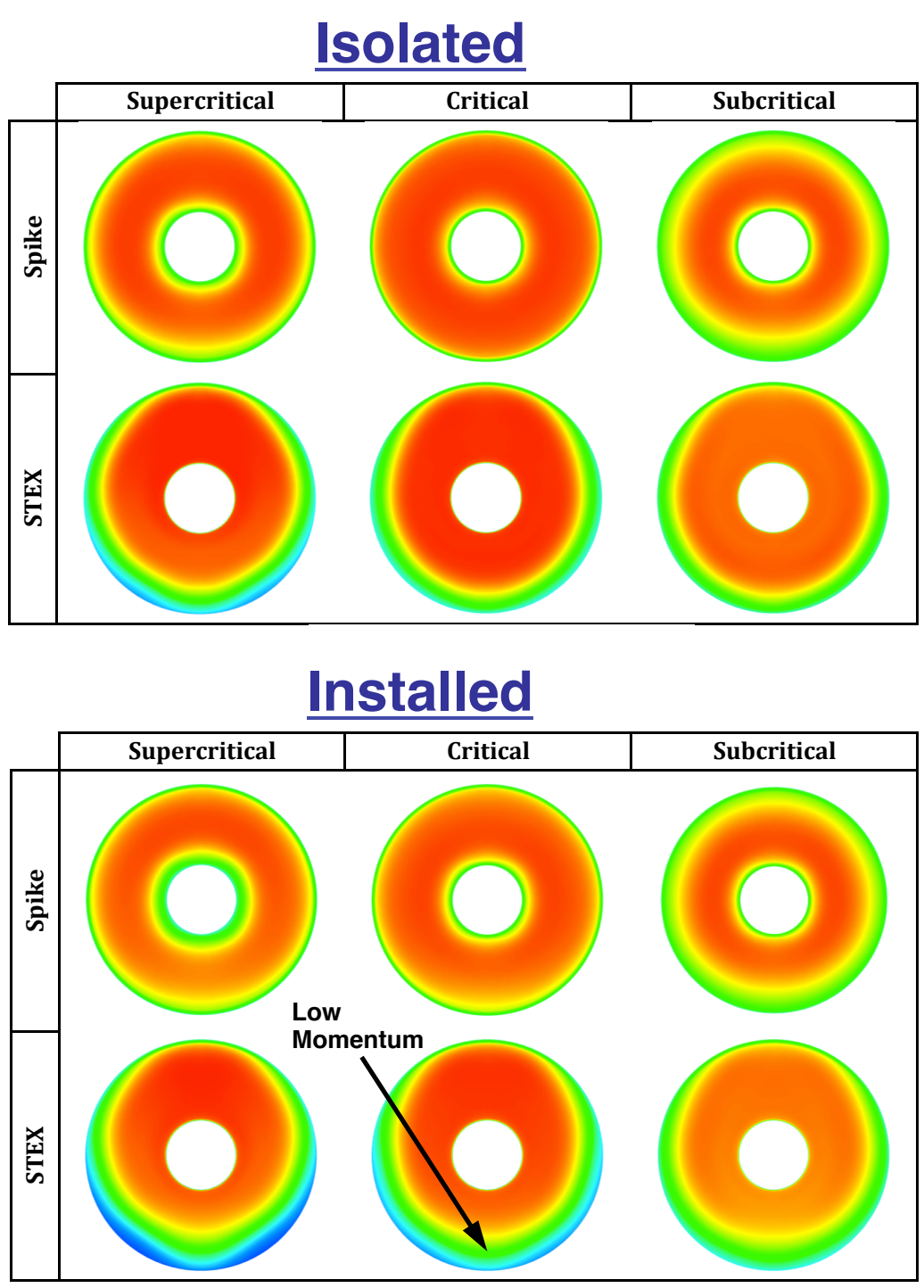

$$
\mathrm{P}_{\mathrm{t}, 2} / \mathrm{P}_{\mathrm{t}, 0} \quad \begin{array}{cccccc}
0.75 & 0.8 & 0.85 & 0.9 & 0.95 & 1
\end{array}
$$




\section{Vehicle Performance Comparison}

High pressure on

Spike spike nacelle
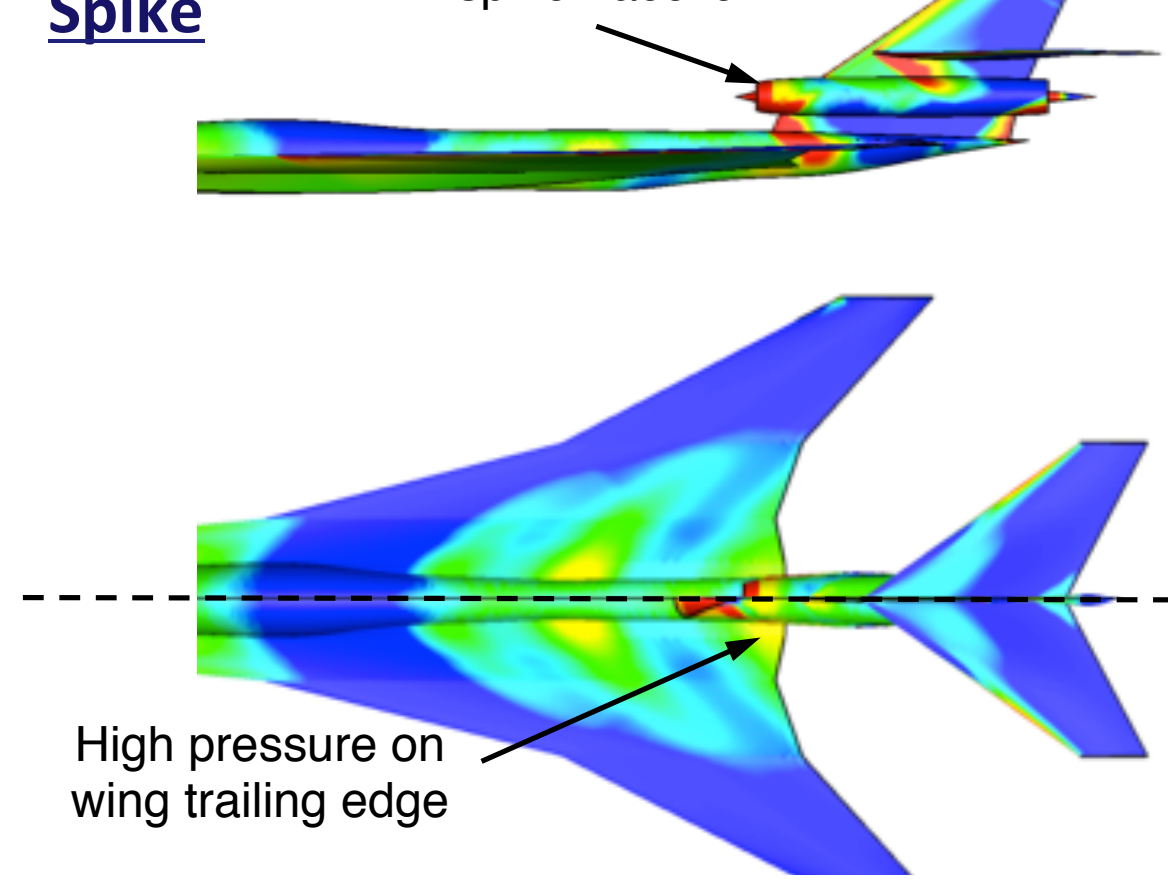

STEX Low pressure on
STEX nacelle

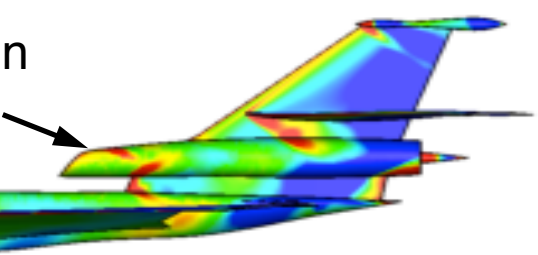

\begin{tabular}{|c|c|c|}
\hline Parameter & Spike & STEX \\
\hline $\mathrm{a}\left(^{\circ}\right)$ & 3.26 & 3.23 \\
\hline Airframe L/D & 4.75 & 4.94 \\
\hline $\mathrm{D}_{\text {net }}(\mathrm{lbf})$ & 4391 & 4230 \\
\hline TSFC (lbm/lbf-hr) & 1.452 & 1.416 \\
\hline Range & - & $+6.6 \%$ \\
\hline
\end{tabular}




\section{Vehicle Sonic Boom Comparison}

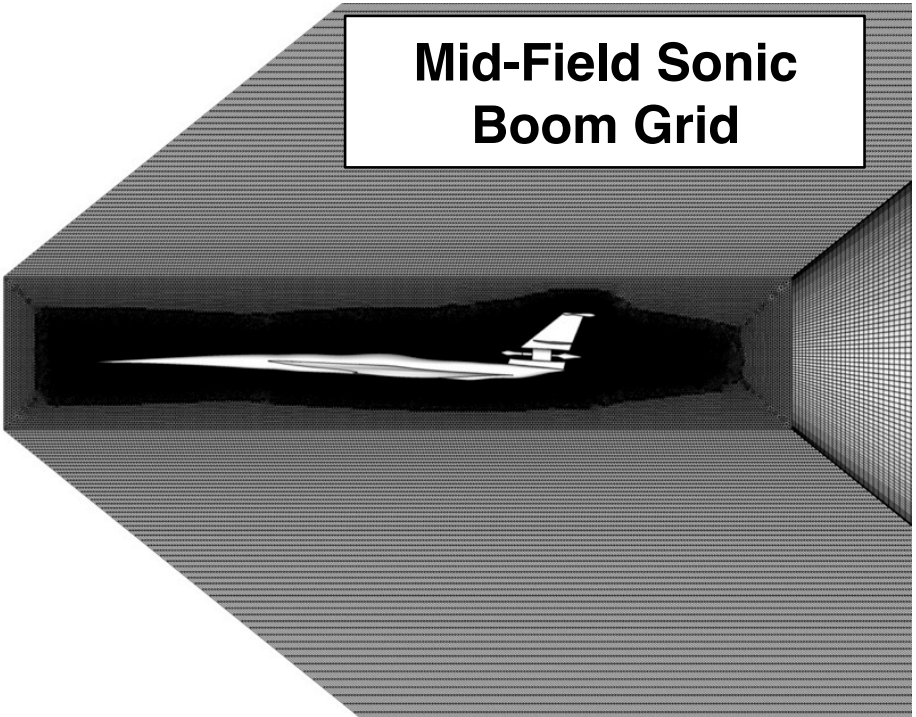

- Mach-aligned extruded prism grid generated using Inflate out to 6 body lengths

- Pressure signals extracted from $\mathrm{h} / \mathrm{L}=1-5$ at $\Phi=0^{\circ}-50^{\circ}$

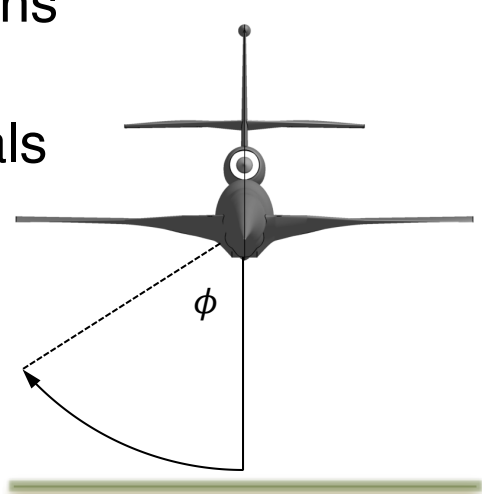

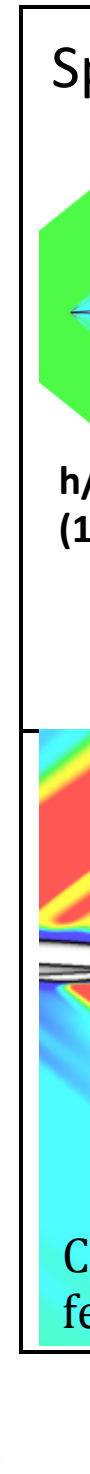

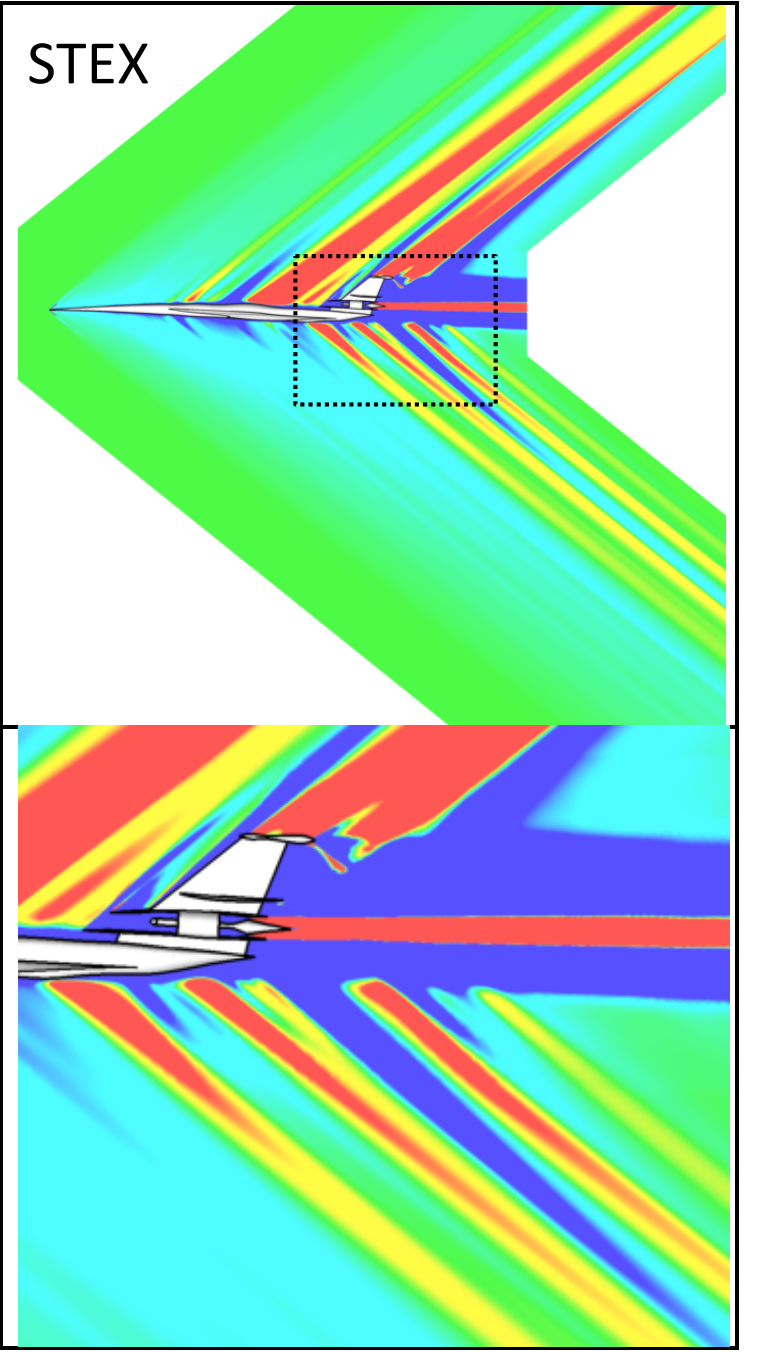

Mach \#

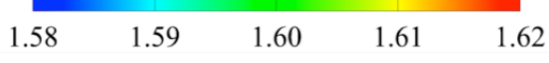




\section{Mid-Field Pressure Waveform Comparison}
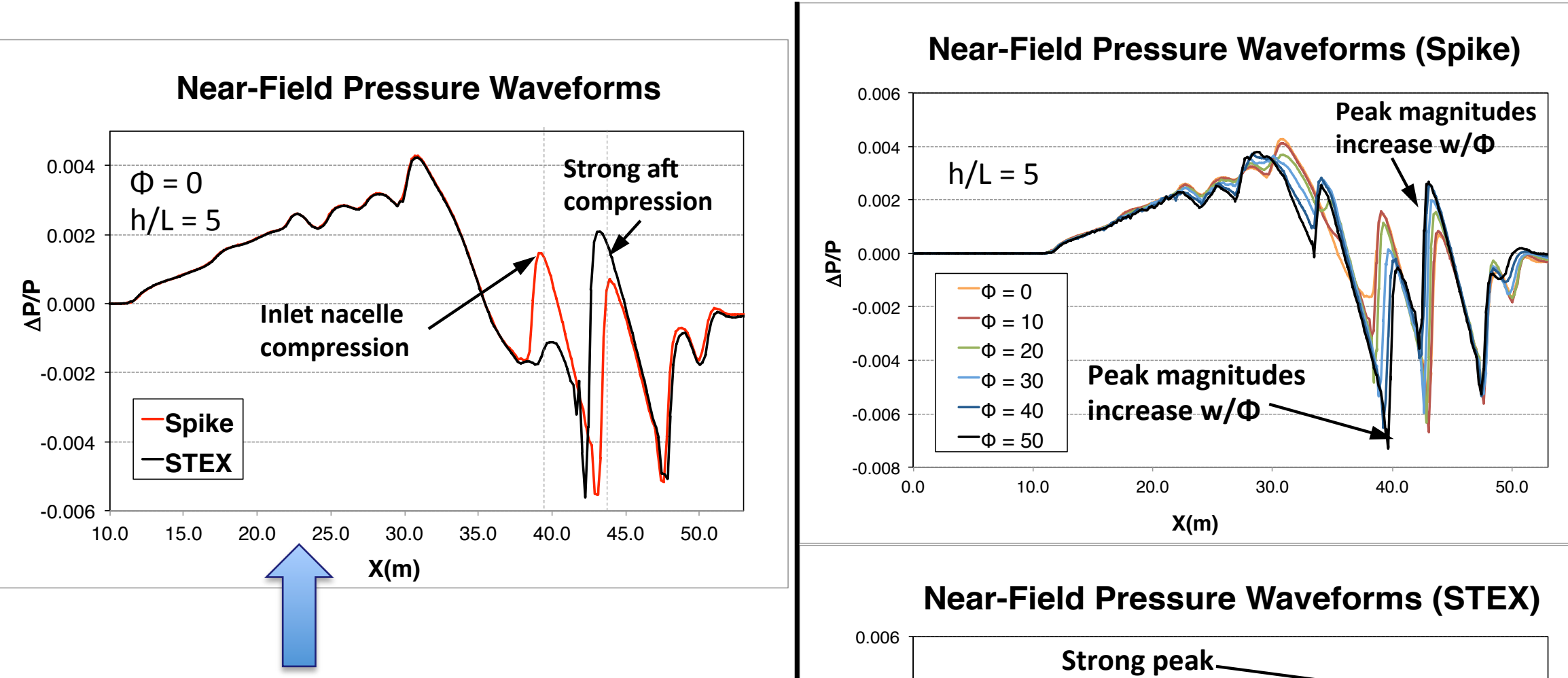

\section{Under-track}

Near-Field Pressure Waveforms (STEX)

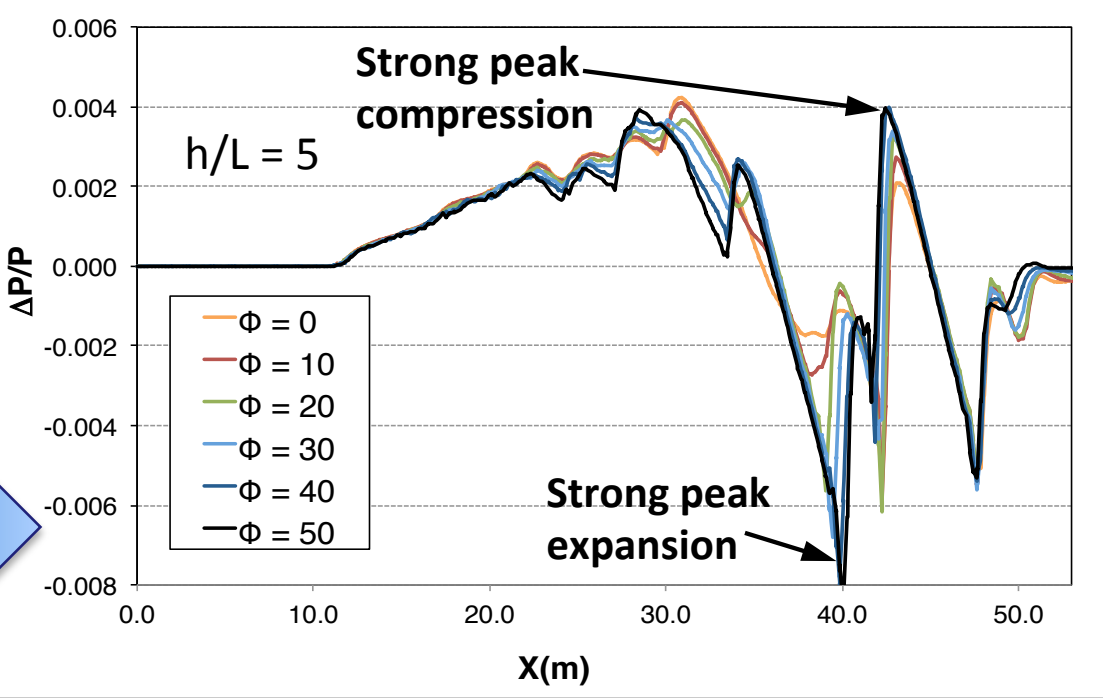




\section{Vehicle Propagated Ground Signature Comparison}

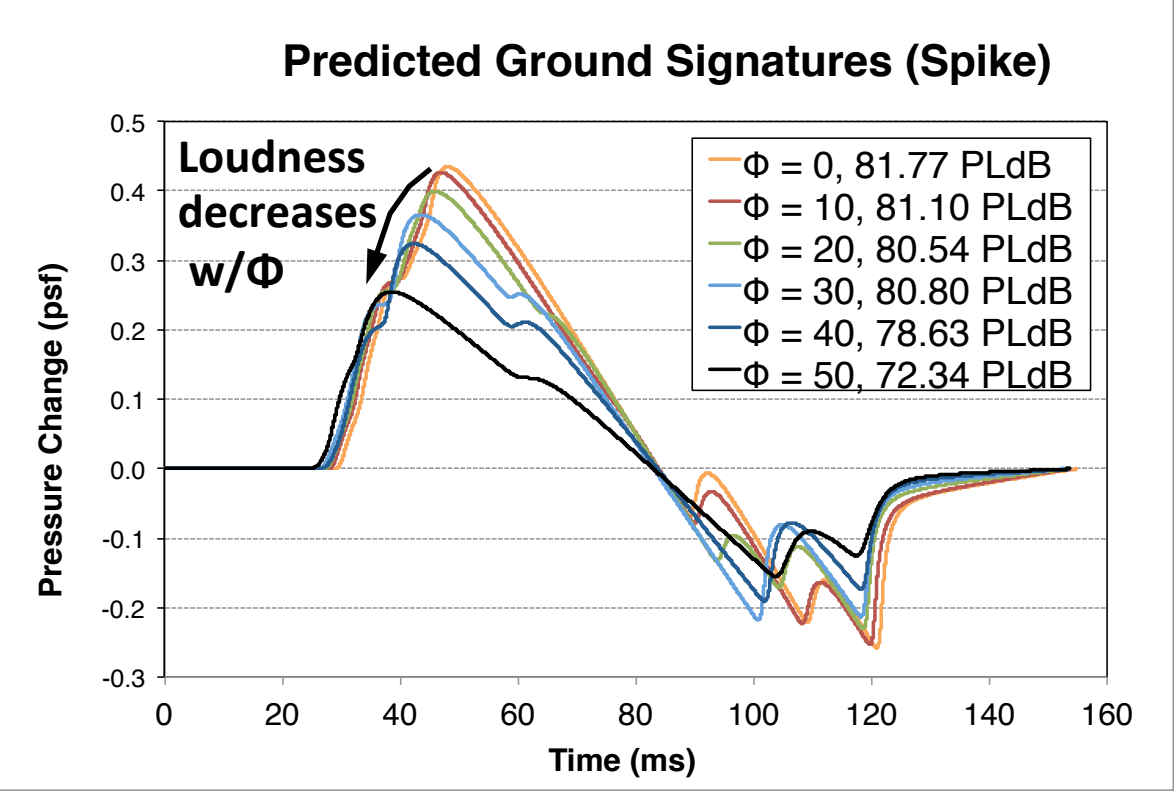

Predicted Ground Signatures (STEX)

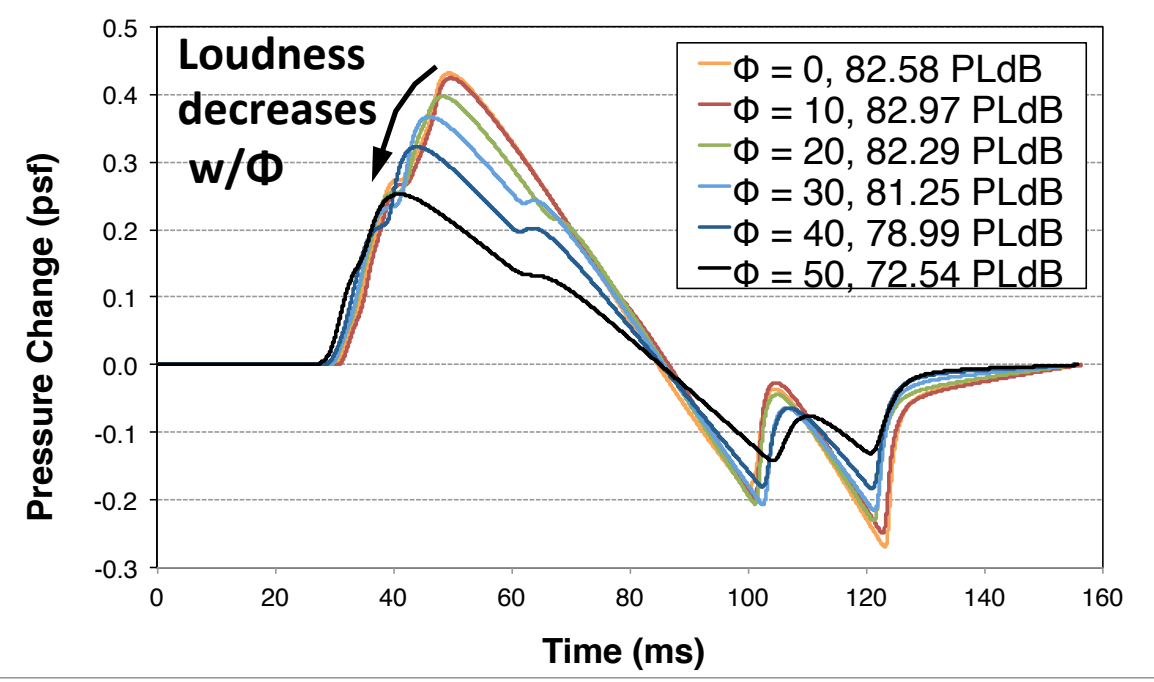

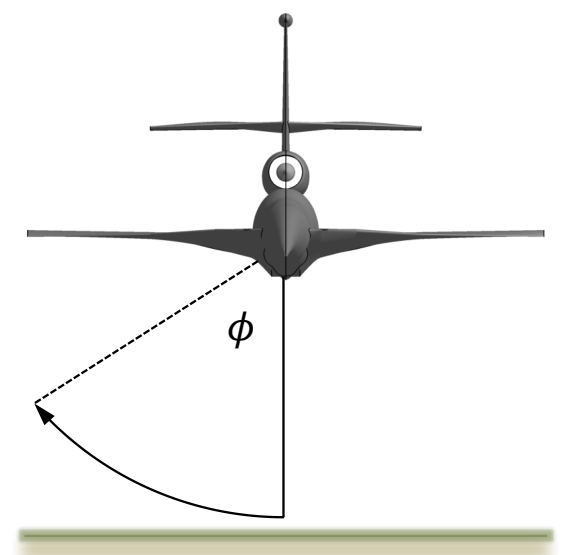

- Under-track loudness higher than original design ( 82 vs. 76.4 PLdB)

- Differing engine geometry

- Euler vs. RANS (viscous effects)

- Re-adjusted $\alpha$ to hit target $C_{L}$

- Adjoint-adapted grids vs. geometry refined

- Improvement to sonic boom performance likely recoverable with additional RANS aerodynamic shaping 


\section{Conclusions}

$>$ Inlet trade study conducted to capture effects of engine installation on inlet performance

$>$ Simultaneously captured the effects of engine installation on aircraft performance AND sonic boom 


\section{Conclusions}

Spike inlet configuration:

$>\sim 3 \%$ higher total pressure recovery

$>>70 \%$ lower inlet distortion

$>\sim 1 \%$ lower propagated ground loudness

STEX inlet configuration:

$>$ Lower external wave drag ( 160-lbf)

$>\sim 4 \%$ higher vehicle L/D ratio

$>\sim 2.5 \%$ lower TSFC

$>+6.6 \%$ increased range capability 


\section{Conclusions}

$>$ Integration of a "low-boom" inlet does not automatically guarantee reduction in overall vehicle sonic boom signature.

$>$ Inlet interaction with the vehicle signature plays a much more dominant role.

$>$ Inlet integration should be considered during the conceptual vehicle design optimization process. 


\section{Acknowledgements}

NASA's Commercial Supersonic Technology (CST) Project

Jon Seidel - Thermodynamic cycle support

Chuck Trefny - Propulsion-airframe integration

Bob Haimes - SUPIN and ESP software integration

John Dannenhoffer - ESP custom parameterizations 
